\title{
High Dynamic Pressures and Modest Temperatures: A Broad Perspective and Bridging the Gap
}

\author{
W. J. Nellis
}

This article was submitted to

$18^{\text {th }}$ International Conference on High Pressure Science and

Technology

Bejing, China

July 23-27, 2001

U.S. Department of Energy

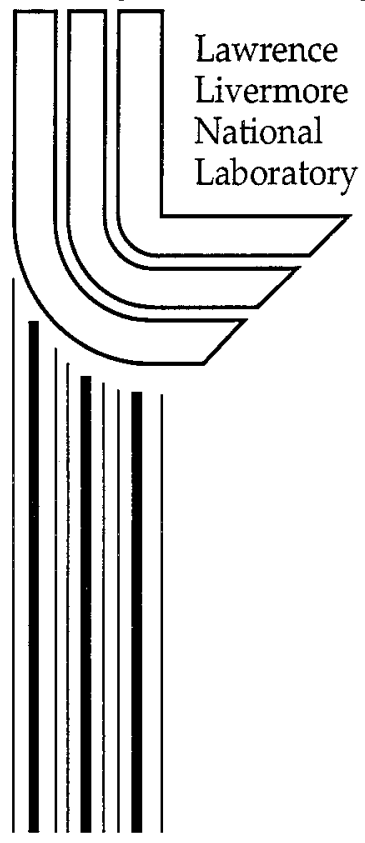

August 30, 2001 


\section{DISCLAIMER}

This document was prepared as an account of work sponsored by an agency of the United States Government. Neither the United States Government nor the University of California nor any of their employees, makes any warranty, express or implied, or assumes any legal liability or responsibility for the accuracy, completeness, or usefulness of any information, apparatus, product, or process disclosed, or represents that its use would not infringe privately owned rights. Reference herein to any specific commercial product, process, or service by trade name, trademark, manufacturer, or otherwise, does not necessarily constitute or imply its endorsement, recommendation, or favoring by the United States Government or the University of California. The views and opinions of authors expressed herein do not necessarily state or reflect those of the United States Government or the University of California, and shall not be used for advertising or product endorsement purposes.

This is a preprint of a paper intended for publication in a journal or proceedings. Since changes may be made before publication, this preprint is made available with the understanding that it will not be cited or reproduced without the permission of the author.

This report has been reproduced directly from the best available copy.

Available to DOE and DOE contractors from the Office of Scientific and Technical Information

P.O. Box 62, Oak Ridge, TN 37831

Prices available from (423) 576-8401

http://apollo.osti.gov/bridge/

Available to the public from the National Technical Information Service

U.S. Department of Commerce 5285 Port Royal Rd., Springfield, VA 22161 http://www.ntis.gov/

OR

Lawrence Livermore National Laboratory Technical Information Department's Digital Library http://www.llnl.gov/tid/Library.html 


\title{
High Dynamic Pressures and Modest Temperatures: A Broad Perspective and Bridging the Gap
}

\author{
W. J. Nellis \\ Lawrence Livermore National Laboratory, University of California, Livermore, California 94550 , \\ USA
}

Pressures up to a few $100 \mathrm{GPa}$ and temperatures as high as a few $1000 \mathrm{~K}$ have been achieved with high dynamic pressures using a two-stage light-gas gun. Results are reviewed for molecular fluids, metallic hydrogen, solids, implications for planetary interiors, and structures and properties of materials recovered intact from high dynamic pressures.

Key words: Dense fluids, metallic hydrogen, planetary interiors

\section{Introduction}

Dynamic high pressures are applied rapidly to materials to increase density and temperature, to alter crystal structure and microstructure, and to change physical and chemical properties. Pressures from 1 to $500 \mathrm{GPa}$ (5 Mbar), compressions up to fifteen fold greater than initial liquid density in the case of hydrogen, and temperatures ranging from room temperature up to several ev $(11,600 \mathrm{~K})$ can be achieved by shock compression of condensed matter. In fact, above $1 \mathrm{GPa}$ the terms shock and dynamic are used interchangably. At these extreme conditions the bonding, structure, physical and chemical properties of condensed matter are changed substantially from what they are at ambient. If high pressure phases could be quenched to ambient, then new opportunities become available in condensed matter and material sciences, as well as potential technological applications. The purpose of this paper is to review results obtained with dynamic high pressures achieved in molecular fluids, metallic hydrogen and solids, discuss implications for giant planets, and describe the structure and properties of materials recovered intact from dynamic high pressures.

\section{Molecular Fluids and Metallic Hydrogen}

Perhaps, the most remarkable observation with this method is metallic fluid hydrogen [1-4]. A metallic state of hydrogen had been sought previously for decades. Those experiments used cryogenic liquid specimens at atmospheric pressure because of the high density of the liquid compared to the gaseous phase. The high initial density of the liquid causes a substantially lower temperature and higher density in the shock-compressed state, than if a gaseous sample were used. To enable systematic study of highly compressed fluids, a cryogenic system was developed to synthesize liquid samples [5,6] for compression with a two-stage light-gas gun [2]. This cryogenic sample holder enabled investigations of a wide variety of liquids with initial temperatures ranging from 4 to $230 \mathrm{~K}$, including $\mathrm{He}$ [7], $\mathrm{H}_{2} / \mathrm{D}_{2}$ [1-4,6,8,9], $\mathrm{N}_{2}$ [5,10-13], $\mathrm{O}_{2}$ [5,14,15], $\mathrm{Ar}$ [5], $\mathrm{CH}_{4}$ [16-18], $\mathrm{Xe}$ [19], $\mathrm{CO}$ [16], $\mathrm{CO}_{2}$ [20], and $\mathrm{NH}_{3}[17,21]$ Similar sample holders at room temperature were used to study $\mathrm{H}_{2} \mathrm{O}$ [2124], synthetic Uranus (a mixture of water, ammonia, and isopropanol) $[17,25,26]$ and $\mathrm{CH}_{2}$ and $\mathrm{C}_{6} \mathrm{H}_{6}$ $[18,27,28]$.

Because of the short lifetime of a shock experiment, the compression is adiabatic and temperature increases. The lifetime of a shock state is $\sim 100 \mathrm{~ns}$ for sample dimensions typical of twostage-gun experiments. This time is more than sufficient to achieve thermal equilibrium in dense fluids. The actual temperature achieved depends sensitively on the number of shocks used to compress to a given pressure. The greater the number of shocks, the lower the temperature and higher the density. High dynamic pressures are generated by impact of a planar impactor onto the sample holder. The impactor is accelerated to velocities in the range 1 to $8 \mathrm{~km} / \mathrm{s}$ with a two-stage light-gas gun. The Hugoniot equation of state (EOS) is the locus of pressure-volume-energy (P-V-E) states achieved by single shock compression. The three Hugoniot equations are based simply on conservation of momentum, mass, and internal energy across a shock front. Hugoniot points are obtained by measuring impactor velocity and shock velocity, using the previously measured Hugoniot EOSs of the 
solid impactor and wall of the liquid layer, and using the shock impedance match principal, which states that shock pressure and material velocity are continuous across an interface between two dissimilar materials. Temperature is measured from the optical spectrum emitted from a shock front. Electrical conductivity is measured by inserting electrodes into contact with a shock-compressed sample. Raman spectra are measured by scattering light from a shock-compressed sample. Illustrations of pressure-volume Hugoniot EOS, temperature, and electrical conductivity experiments are shown in Fig. 1. The impactor launched from the gas gun is incident from the left and electrical or optical signals exit to the right.

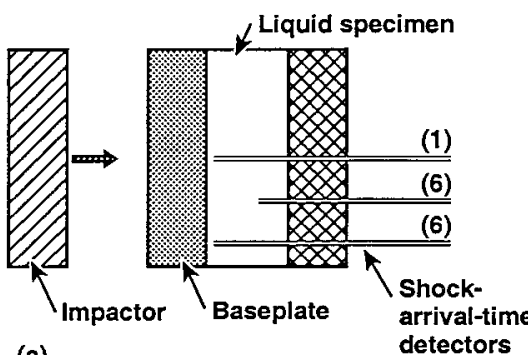

(a)

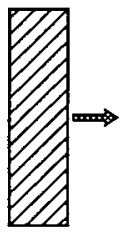

(b)

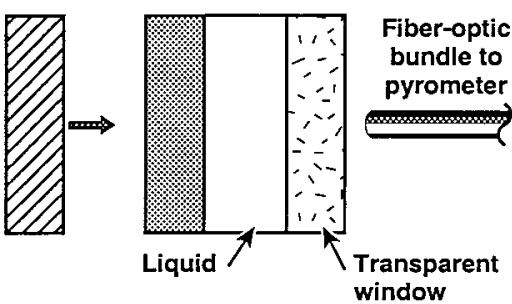

(c)

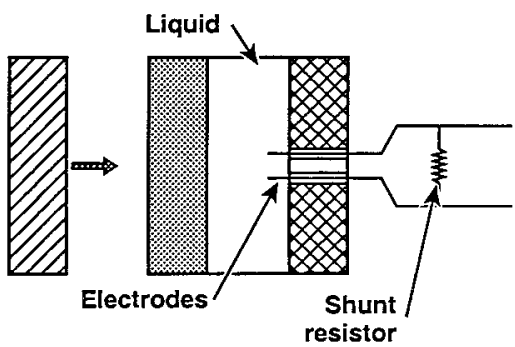

(d)

Fig. 1. Experimental arrangements in which either the first shock or its reflection from an anvil is diagnosed: (a) Single-shock Hugoniot EOS. Shock velocity is measured in liquid by placing detectors on each of two levels; (b) Double-shock Hugoniot EOS. Shock velocity is measured across anvil; (c) Shock emission temperatures under single and double shock (first shock reflects off window to produce second shock); and (d) Electrical conductivity of singly-shocked fluid.

One of the most interesting systems observed with these techniques is the continuous dissociative phase transition in dense fluid nitrogen at 30 to $80 \mathrm{GPa}$ on the Hugoniot $[5,10-12]$. This transition causes two unexpected phenomenon: (i) shock-induced cooling, the process in which the temperature of the shock reflected from the anvil, the second shock, is actually lower than the temperature of the shock incident on the anvil, the first shock, and (ii) temperature decreases with increasing pressure at constant volume. In the case of nitrogen item (ii) is indicative of a large negative Gruneisen parameter, which is challenging to understand $[29,30]$.

The experimental configuration to measure electrical conductivities of multiply shocked hydrogen and other liquids $[1,13,15,24]$ is illustrated in Fig. 2. The shock reverberates in the liquid between the two stiff sapphire anvils. This experiment was used to demonstrate that fluid hydrogen achieves the minimum electrical conductivity of a disordered metal at $140 \mathrm{GPa}$, ninefold compression of initial liquid density, and $3000 \mathrm{~K}$. The finite temperature produced by dynamic compression causes a disordered liquid which facilitates bridging the mobility gap to achieve a metallic state at a lower pressure than expected for the ordered solid. The metallic state is achieved because pressure reduces the $15 \mathrm{ev}$ gap and thermal disorder fills it in until $\mathrm{E}_{\mathrm{g}} / \mathrm{kB}_{\mathrm{B}}=\mathrm{T} \sim 2600 \mathrm{~K}$, where $\mathrm{E}_{\mathrm{g}}$ is smeared out thermally 
and the electronic system has a Fermi surface $\left(E_{g}=19 \mathrm{ev}\right)$. Since $\mathrm{T} / \mathrm{T}_{\mathrm{F}} \sim 0.01$, metallic hydrogen is highly degenerate. Fluid hydrogen becomes metallic via a continuous transition from semiconductor to metal.

Tight-binding MD [31] shows that metallic hydrogen at $3000 \mathrm{~K}$ has a peak in the protonproton pair distribution function at the separation distance between protons in the molecule and that "molecules" or dimers are short-lived $\left(\sim 10^{-14} \mathrm{~s}\right)$. Kinetic, vibrational, and rotational energies of the transient pairs are comparable. Conduction electrons have a very short mean free path, the distance between adjacent particles $(\sim 2 \mathrm{~A})$. This is a strong-scattering system characteristic of minimum metallic conductivity.

Fluid $\mathrm{Cs}, \mathrm{Rb}$, and $\mathrm{H}$ at $\sim 2000 \mathrm{~K}$ metallize with a conductivity of $2000(\Omega-\mathrm{cm})^{-1}$ at the same scaled density $\mathrm{D}_{\mathrm{m}} 1 / 3_{\mathrm{a}}{ }^{*}=0.38$, where $\mathrm{D}_{\mathrm{m}}$ is the density of atoms at metallization and $\mathrm{a}^{*}$ is the effective Bohr radius [32]. This nonmetal-metal transition is a Mott transition.

The Herzfeld criterion, which depends only on polarization of the atom [33], gives a metallization density of $0.60 \mathrm{~mol} \mathrm{H} / \mathrm{cm}^{3}$, within $7 \%$ of $0.64 \mathrm{~mol} \mathrm{H} / \mathrm{cm}^{3}$ determined by fitting the conductivity data to a thermal activation model in the semiconducting regime and determining the density at which the mobility gap is approximately equal to the thermal energy, $\mathrm{E}_{\mathrm{g}} \sim \mathrm{kT}[2]$. At this condition the mobility gap is smeared out thermally and the system has a Fermi surface. This agreement in metallization density between the two methods and the very short short dimer lifetimes $\left(\sim 10^{-14} \mathrm{~s}\right)$ suggest that fluid metallic hydrogen is essentially monomeric.

Metallic conductivity, $2000(\Omega-\mathrm{cm})^{-1}$, is the minimum conductivity of a metal $\sigma_{\min }=2 \pi \mathrm{e}^{2}$ / (3hd), where $h$ is Planck's constant and $d=D_{m}{ }^{-1 / 3}$. At metallization density, $0.60 \mathrm{~mol} \mathrm{H} / \mathrm{cm}^{3}, \sigma_{\min }$ $=6000(\Omega-\mathrm{cm})^{-1}$, in good agreement with experiment. Metallic conductivities in good agreement with experiment were calculated with the Ziman model [4] and with tight-binding molecular dynamics [34]. The latter show that the nonmetal-metal transition in hydrogen is density-driven.

In contrast, to the systematic Mott transition to a metallic state in fluid hydrogen [1], oxygen [15], and nitrogen [13], water is a proton conductor at $100 \mathrm{GPa}$ pressures [24].

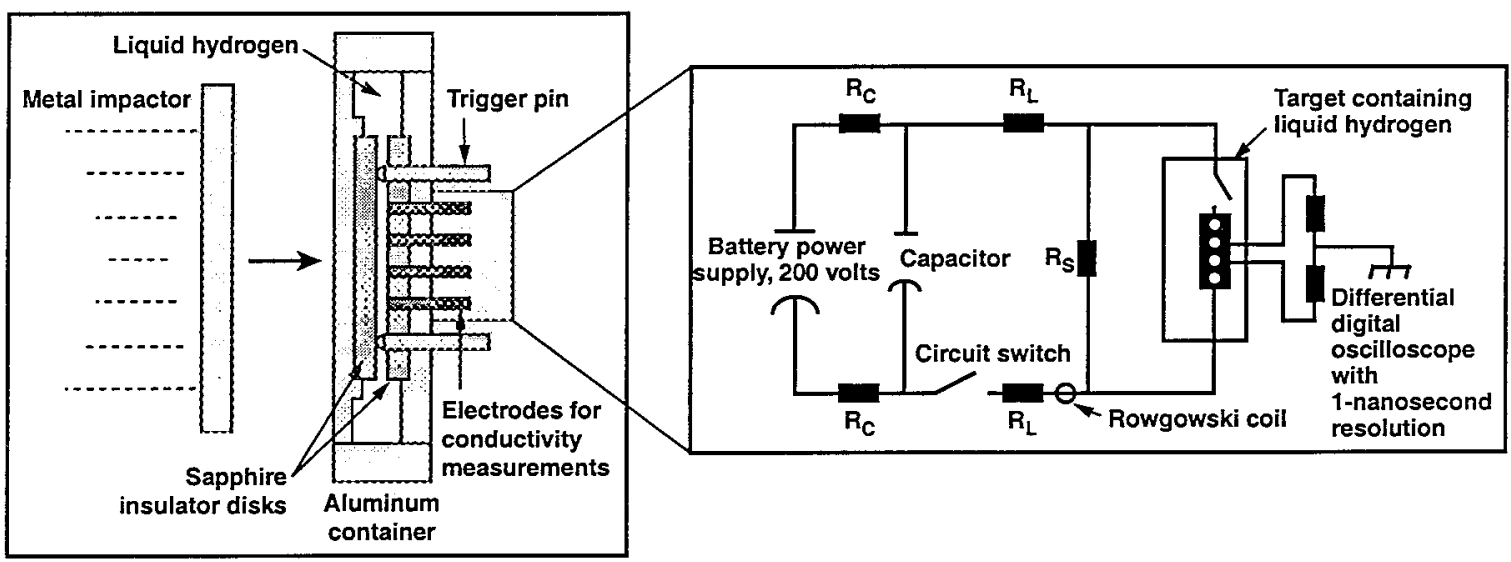

(a)

(b)

Fig. 2. Schematic of electrical conductivity experiment using shock wave reverberating between stiff sapphire anvils. Four electrodes in (a) were connected to circuit in (b). For conductivities lower than metallic, two probes were used. Impactor is accelerated to $\sim 7 \mathrm{~km} / \mathrm{s}$ with two-stage gun [2].

\section{Solids}

In order to perform experiments on fluids, it is necessary to know the Hugoniot EOS of the solids used as impactors and as walls of the sample holder. For this reason the Hugoniots of the metals Al, 
$\mathrm{Cu}$, and Ta were measured up to a few $100 \mathrm{GPa}$ with a two-stage light gas gun [35]. Higher pressures were measured in various solid specimens using underground nuclear explosives [36-38].

The graphite to diamond transition was time-resolved with a velocity interferometer for a surface of any reflector (VISAR) $[39,40]$. These experiments showed that the dynamics of this transition are sensitive to the direction of shock propagation with respect to crystallographic orientation and to the microstructure of the sample.

\section{Giant Planets: Experimental Constraints}

The Jovian planets Jupiter and Saturn together contain over 400 Earth masses, most of which is hydrogen. Pressure and temperature in the mantle of Jupiter range up to a $300 \mathrm{GPa}$ and several 1000 $\mathrm{K}$ and are about $4 \mathrm{TPa}$ and $20,000 \mathrm{~K}$ at the center [41]. Hydrogen is fluid at these conditions [42]. Magnetic fields of giant planets are produced by the convective motion of electrically conducting fluid hydrogen by dynamo action [43]. These conditions raise some interesting questions about Jupiter. For example, why is the magnitude of the Jovian magnetic field so large and asymmetric relative to that of Earth and is there a relatively sharp core-mantle boundary in Jupiter between a molecular mantle and monatomic core, analogous to the boundary in the Earth between the rocky mantle and iron core? The most important material to study with respect to the Jovian planets is hydrogen because it has by far the greatest cosmological abundance. The most important materials to study with respect to the "icy" planets Uranus and Neptune are water, ammonia, methane, and synthetic Uranus, a representative mixture. The purpose of this section is to review the current experimental situation for hydrogen, water, ammonia, methane, and synthetic Uranus and to describe implications for the nature of the interiors of the Jovian and "icy" planets [44-47]

The transition from diatomic insulating $\mathrm{H}_{2}$ to monatomic metallic $\mathrm{H}$ at high pressures and temperatures in the fluid has been an important issue in Jovian modeling for decades. Some theoretical work suggested that this transition, often called the plasma phase transition (PPT), is first order [4850]. Other work suggested that this transition is continuous in pressure and temperature [51]. Since at high shock pressures (>15 GPa) and temperatures, no first-order phase transition has ever been observed in any fluid, it is quite likely that this transition is continuous and, thus, it is most likely that no sharp core-mantle boundary exists in the Jovian planets.

Depending on the model, the radius in Jupiter at which metallization occurs has ranged between 0.75 and $0.90 R_{J}$, where $R_{J}$ is the radius of Jupiter. We estimated where the Jovian magnetic field is made by estimating where the electrical conductivity of dense fluid hydrogen is large. For purposes of discussion we assume that the path of pressure-temperature (P-T) states in Jupiter is an isentrope. Using a fluid model based on our equation-of-state data, the isentrope of pure hydrogen was calculated from the surface temperature of Jupiter $(165 \mathrm{~K})$ [44]. Since He is a small molecule as hydrogen, the equation of state of a mixture containg $\sim 10$ at.\% He is not expected to be substantially different from that of pure hydrogen. Temperature rises steeply with increasing pressure (depth in Jupiter) until molecular dissociation begins at $\sim 40 \mathrm{GPa}$. At higher pressures up to $\sim 200 \mathrm{GPa}$ temperature varies slowly because internal energy is absorbed in dissociation. Electrical conductivity was calculated along the Jovian isentrope using a scaling relationship derived from measured conductivities [45]. Metallization (2000 $\left.(\Omega-\mathrm{cm})^{-1}\right)$ probably occurs in Jupiter at $140 \mathrm{GPa}$ and $4000 \mathrm{~K}$, as it does in our laboratory experiments at $140 \mathrm{GPa}$ and $3000 \mathrm{~K}$, because electrical conductivity is generally slowly varying with temperature in a disordered liquid metal. A pressure of $140 \mathrm{GPa}$ is reached in Jupiter at a radial position of $\sim 0.90 \mathrm{R}_{\mathrm{J}}$. Assuming that material with a conductivity as low as that in Uranus, $20(\Omega-\mathrm{cm})^{-1}$, contributes to the Jovian surface field, then radii out to $95 \% \mathrm{R}_{\mathrm{J}}$ contribute to the surface magnetic field. The model for Jupiter derived from these considerations is illustrated in Fig. 3. 

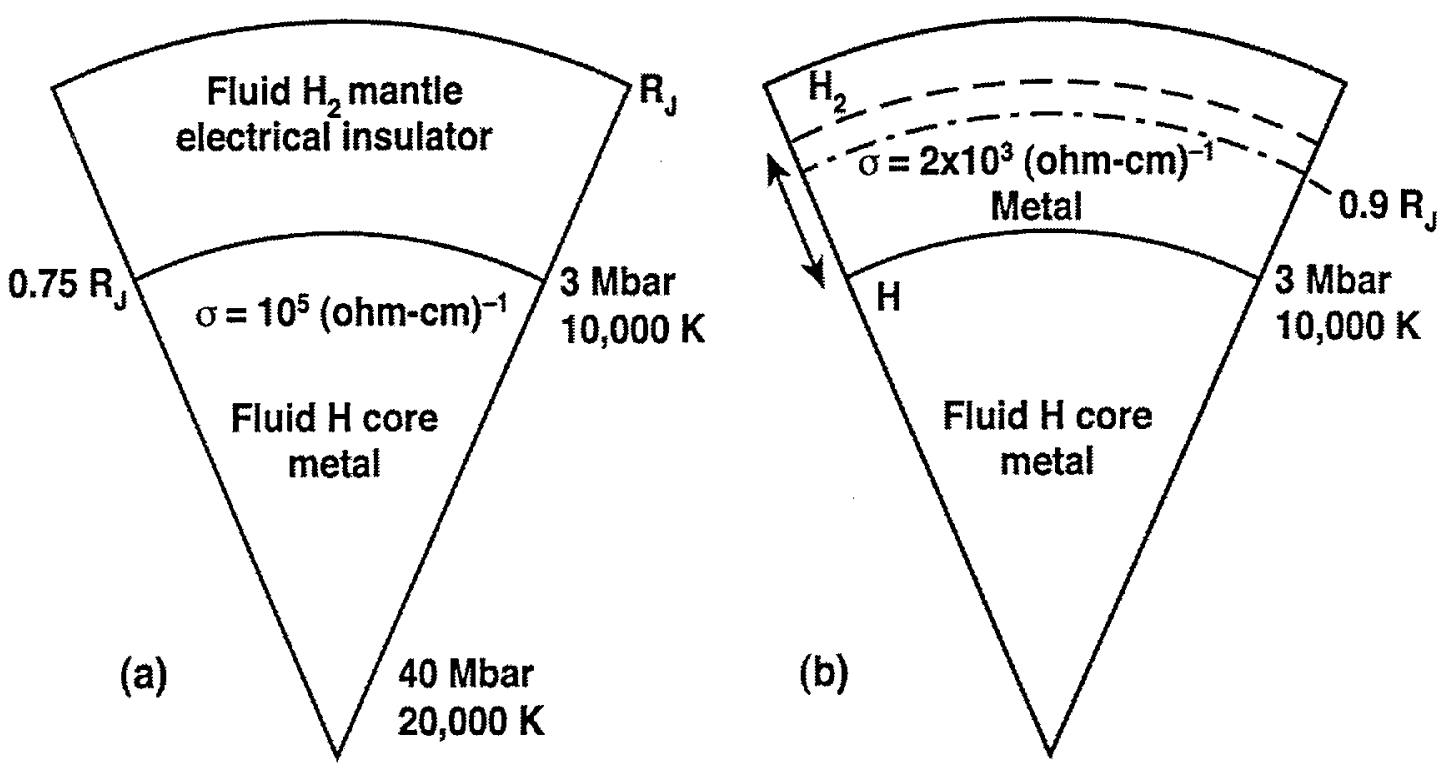

Figure 3. Schematics of Jovian interior (a) before and (b) after recent hydrogen experiments at high dynamic pressures and temperatures comparable to those in Jupiter. Previous picture in (a) has insulating molecular mantle which transitions to monatomic metallic core via first-order phase transition at $0.75 \mathrm{R}_{\mathrm{J}}$. Picture in (b) shows that $\mathrm{H}_{2}$ is molecular down to $\sim 40 \mathrm{GPa}$ at $\sim 0.95 \mathrm{R}_{\mathrm{J}}$, (long-dashes), at which depth dissociation commences and is completed by $\sim 0.75 \mathrm{R}_{\mathrm{J}}$. (solid curve). Metallization occurs at a depth of at $\sim 0.90 \mathrm{R}_{\mathrm{J}}$ (dot-dashes) [45].

Although surface magnetic fields decrease with distance from where they are produced, the Jovian surface magnetic field is probably not significantly lower than where the field is produced because it is generated so close to the surface and, thus, its magnitude is relatively large, $\sim 10 \mathrm{G}$. The large asymmetry in the surface magnetic field is also readily observed for the same reason [52]. That is, planetary magnetic fields are typically composed of dipolar and higher order components. These multipole components are proportional to $\mathrm{r}^{-\mathrm{n}}$, where $\mathrm{n}=3,4,5, \ldots$ The deeper the magnetic field is produced, the smaller are components of the surface field caused by higher order multipole components and the more symmetric is the magnetic field.

In contrast with Jupiter, in Saturn the metallic phase of hydrogen is reached at $\sim 0.5 R_{S}$, where $R_{S}$ is the radius of Saturn [53], much deeper in the planet than in Jupiter. The equatorial surface magnetic field of Saturn is $0.21 \mathrm{G}$, substantially smaller than the equatorial surface magnetic field of Jupiter. Thus, the fact that the magnetic field of Jupiter is generated close to the surface and in Saturn it is generated much deeper is consistent with the relative magnitudes of their external magnetic fields.

The planets Uranus and Neptune are thought to have evolved from the accretion of nebular water, ammonia, and methane. At high pressures and temperatures these molecules react chemically to form complex mixtures. The EOSs of these mixtures are responsible for mass distributions and, hence, gravitational moments and their electrical conductivities are responsible for magnetic fields of the icy planets. Water is one of the most studied fluids at high shock pressures. Recent work includes Hugoniot EOS [21], shock temperatures [22], Raman spectroscopy [23], and electrical conductivities $[21,24]$. The Hugoniot [21], shock temperatures [17], and electrical conductivities [21] of ammonia have been measured. The Hugoniot EOS [16,28,54], shock temperatures [17], and electrical conductivities [18] of methane and other hydrocarbons have been measured. The mixture "synthetic Uranus" is composed of water, ammonia, and isopropanol in proportions which give nearcosmological abundance ratios of $\mathrm{H}, \mathrm{O}, \mathrm{C}$, and $\mathrm{N}$. Hugoniot, temperature, and conductivity data have been measured $[17,25,26,55]$. These data suggest a maximum conductivity of $20(\Omega-\mathrm{cm})^{-1}$ inside the giant icy planets, which is responsible for their magnetic fields. 


\section{Materials Synthesized and Recovered from High Dynamic Pressures}

Dynamic high pressures are used to produce novel crystal structures, microstructures, and associated properties by subjecting specimens to dynamic pressures and recovering them intact. Pressure is applied at strain rates up to $10^{8} / \mathrm{s}$ and higher, pressures and temperatures reach as high as $100 \mathrm{GPa}(1 \mathrm{Mbar})$ and a few $1000 \mathrm{~K}$ for $\sim \mu \mathrm{sec}$, and quench at rates up to $10^{12} \mathrm{bar} / \mathrm{s}$ and $10^{9} \mathrm{~K} / \mathrm{s}$, as illustrated in Fig. 4. While pressure releases to zero, temperature releases to a residual value, which is higher than the initial value because of irreversible shock heating. The residual temperature approaches the ambient value by thermal conduction into surrounding material. Such experiments are called recovery experiments. The high quench rates mean that phases and structures synthesized at high shock pressures and temperatures might be quenched to ambient.

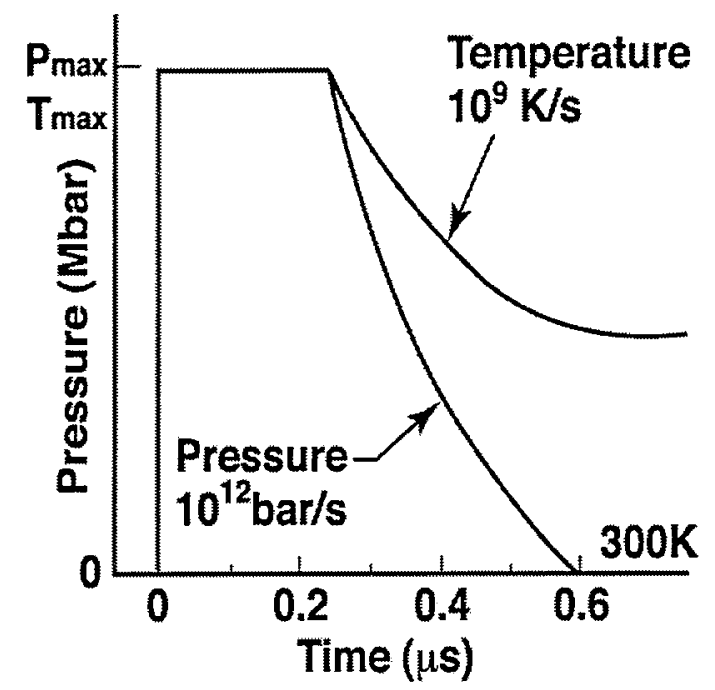

Fig. 4. Shock and release profiles calculated in planar geometry for shockcompressed $\mathrm{Cu}$. Pressure releases to zero but temperature does not because of irreversible shock-heating.

Experiments discussed here use a two-stage light-gas gun [56] to accelerate a planar metal projectile to a maximum impactor velocity of $\sim 4 \mathrm{~km} / \mathrm{s}$ with He driving gas, which produces $120 \mathrm{GPa}$ for a $\mathrm{Cu}$ plate impacting a $\mathrm{Cu}$ target. Recovering a sample intact becomes progressively very difficult above $100 \mathrm{GPa}$. Specimens are typically $10 \mathrm{~mm}$ in diameter and 0.001 to $1 \mathrm{~mm}$ thick. Initial temperatures can be varied in the range 100 to $1300 \mathrm{~K}$ [57]. A representative configuration of the fixture which holds a specimen which is shocked and recovered intact is illustrated in Fig. 5. Some examples of effects produced in materials are described below. 


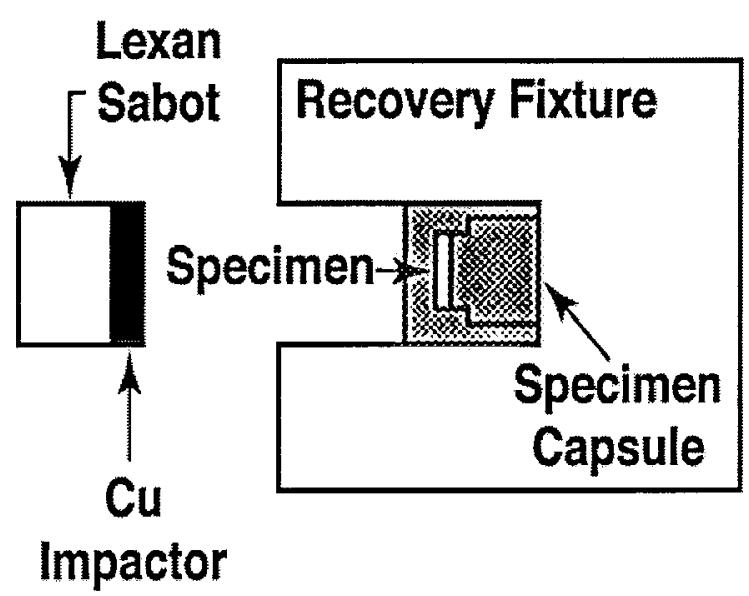

Fig. 5. Schematic of an experiment to recover specimen shocked to pressures up to $100 \mathrm{GPa}$. Projectile is launched by two-stage light-gas gun $[2,56]$.

\subsection{Nanocrystalline Materials}

Shock compression of single-crystal quartz causes the formation of polycrystalline grains, which facilitates flow of the bulk sample under rapid deformation at shock pressures of 20 to $50 \mathrm{GPa}$. As a result the grain boundaries reach very high heterogeneous temperatures, which reduce $\mathrm{SiO}_{2}$ to $\mathrm{Si}$ and $O$, which in turn phase separate. Because the duration of maximum pressure in these experiments is a few $100 \mathrm{~ns}$, there is sufficient time only for nanocrystalline $\mathrm{Si}(\mathrm{n}-\mathrm{Si}$ ) particles to nucleate and grow in situ within the solid $\mathrm{SiO}_{2}$. These nanoparticle have the well-ordered diamond crystal structure [58]. The size distribution of $\mathrm{n}$-Si can be tuned by choice of shock pressure and its duration. The chemical history of this $\mathrm{n}-\mathrm{Si}$ is completely different than that of samples made with traditional wet-chemistry methods, which might shed light on the mechanism of their photoluminescence; that is, is photoluminescence caused by quantum confinement or oxygen passivation of the Si surface, and what are the optical effects caused by shock-induced defects?

\subsection{Films}

High-pressure phases might be synthesized and quenched metastably. $\mathrm{Nb}$ films $1-10 \mu \mathrm{m}$ thick have been recovered from $100 \mathrm{GPa}$ shock pressure [59], demonstrating the feasibility of this process for thin samples with maximum quench rates. This rapid quenching method might be useful, for example, for quenching metallic fluid hydrogen in a one micron-thick sample to a metallic glass at ambient pressure [60]. It has been shown calculationally that pressures ( 140 GPa) and temperatures $(\sim 3000 \mathrm{~K})$ required for hydrogen metallization can be achieved by irradiation with a high-intensity pulsed laser [61].

\subsection{Shock-Induced Defects and Fluxpinning}

Shock deformation induces dislocations and stacking faults, which pin magnetic flux and enhance magnetic hysteresis and magnetic levitation force in superconducting $\mathrm{YBa}_{2} \mathrm{Cu}_{3} \mathrm{O}_{7-\mathrm{x}}$. By using highly ordered melt-textured specimens and orienting their slip plane at $30^{\circ}$ with respect to the shock direction, these brittle oxide specimens can be shocked without macroscopic fracture. After annealing the shocked specimen in oxygen to repair shock-induced damage in bulk, dislocations are converted into stacking faults. As a result the magnetic hysteresis, and thus critical current density, at $1 \mathrm{kOe}$ and $70 \mathrm{~K}$ is about $20 \%$ greater than the value before shock at $7 \mathrm{GPa}$ [62].

Shock compaction of $\mathrm{SmCo}_{5}$ particles at $\sim 8 \mathrm{GPa}$ enhances cohersive forces, increasing the "permanency" of this permanent ferromagnet [63].

\subsection{Synthesis of Hard Materials}

Potentially new hard materials can be synthesized and recovered from dynamic high pressures. For example, shock-compressed mixtures of $\mathrm{C}_{60}$ fullerenes and $\mathrm{Cu}$ powders produce fine 
grained diamond [64]. Nanocrystalline metastable diamond films have been produced by shock compression of $2 \mu \mathrm{m}$ thick $\mathrm{C}_{60}$ fullerene films [65].

\subsection{Powder Consolidation}

Dynamic compaction rapidly consolidates powders by depositing compressive energy on particle surfaces, which are heated heterogeneously, often bond together, and then quench thermally to the interiors of the particles forming a dense compact. Advantages include: i) focussing energy on particle surfaces so that the whole bulk system does not need to be heated for compaction and ii) consolidating powders so quickly that grains do not have time to grow nor do metastable phases have time to decompose. Single-piece disks of both conventional and rapidly solidified alumina/zirconia ceramics have been consolidated by a reverberating shock wave [66]. A computational model has been developed for dynamic compaction which could be applied to a wide variety of materials, pressures, and consolidation rates and, thus, address key issues computationally [67].

\subsection{Shock-Induced Melting and Rapid Resolidification}

Because powders have a lower initial density than a crystalline solid, powders are more compressible and, thus, have higher internal shock energies and higher shock temperatures at a given volume than do solid specimens. Thus, there is a range of relatively high shock pressures in which powders are readily shock melted in bulk and thermally quenched by thermal conduction into surrounding solid metals such as $\mathrm{Cu}$. At relatively lower shock pressures powders are simply compacted dynamically by a shock as discussed in 5.5. Model calculations were used to predict that $\mathrm{Cu}-\mathrm{Zr}$ powders would compact at $16 \mathrm{GPa}$ and shock-melt at $60 \mathrm{GPa}$, as observed by experiment [68].

\section{ACKNOWLEDGMENT}

This work was performed under the auspices of U.S. Department of Energy by the University of California Lawrence Livermore National Laboratory under Contract No. W-7405-ENG-48. 
References

[1] S. T. Weir, A. C. Mitchell, W. J. Nellis, Phys. Rev. Lett., 76, 1860 (1996).

[2] W. J. Nellis, S. T. Weir, A. C. Mitchell, Phys. Rev. B, 59, 3434 (1999).

[3] W. J. Nellis, Scientific American, May, 84, 2000.

[4] W. J. Nellis, A. A. Louis, and N. W. Ashcroft, Phil. Trans. R. Soc. Lond. A, 356, 119 (1998).

[5] W. J. Nellis A. C. Mitchell, J. Chem. Phys., 73, 6137 (1980).

[6] W. J. Nellis et al, J. Chem. Phys., 79, 1480 (1983).

[7] W. J. Nellis et al, Phys. Rev. Lett., 53, 1248 (1984).

[8] W. J. Nellis et al, Phys. Rev. Lett., 68, 2937 (1992).

[9] N. C. Holmes, W. J. Nellis, M. Ross, Phys. Rev. B, 52, 15,835 (1995).

[10] W. J. Nellis et al, Phys. Rev. Lett., 53, 1661 (1984).

[11] H. B. Radousky et al, Phys. Rev. Lett., 57, 2419 (1986).

[12] W. J. Nellis et al, J. Chem. Phys. 94, 2244 (1991).

[13] R. Chau, A. C. Mitchell, and W. J. Nellis (unpublished).

[14] D. C. Hamilton et al, J. Chem. Phys., 88, 5042 (1988).

[15] M. Bastea, A. C. Mitchell, W. J. Nellis, Phys. Rev. Lett., 86, 3108 (2001).

[16] W. J. Nellis et al, J. Chem. Phys., 75, 3055 (1981).

[17] H. B. Radousky et al, J. Chem. Phys., 93, 8235 (1990).

[18] W. J. Nellis, D. C. Hamilton, A. C. Mitchell, J. Chem. Phys., 115, 1015 (2001).

[19] W. J. Nellis, M. van Thiel, A. C. Mitchell, Phys. Rev. Lett., 48, 816 (1982).

[20] W. J. Nellis et al, J. Chem. Phys., 95, 5268 (1991).

[21] A. C. Mitchell W. J. Nellis, J. Chem. Phys., 76, 6273 (1982).

[22] G. A. Lyzenga et al, J. Chem. Phys., 76, 6282 (1982).

[23] N. C.Holmes et al, Phys. Rev. Lett., 55, 2433 (1985).

[24] R. Chau et al, J. Chem. Phys., 114, 1361 (2001).

[25] W. J. Nellis et al, Science, 240, 779 (1988).

[26] W. J. Nellis et al, J. Chem. Phys., 107, 9096 (1997).

[27] D. C. Hamilton et al, J. Chem. Phys., 88, 7706 (1988).

[28] W. J. Nellis et al, J. Chem. Phys., 80, 2789 (1984).

[29] M Ross, J. Chem. Phys., 86, 7110 (1987).

[30] S. Mazevet et al, Los Alamos National Laboratory, private communication (2001).

[31] T. J. Lenosky et al, Phys. Rev. B, 55, R11907 (1997).

[32] F. Hensel and P. Edwards, Phys. World, 4, 43 (1996).

[33] K. F. Herzfeld, Phys. Rev., 29, 701 (1927).

[34] Kress, J., et al. In: Strongly Coupled Coulomb Systems, edited by G. J. Kalman, J. M. Rommel, and K. Blagoev (Plenum, New York,1998), pp. 331-335.

[35] A. C. Mitchell, W. J. Nellis, J. Appl. Phys., 52, 3363 (1981).

[36] W. J. Nellis, J. A. Moriarty, A. C. Mitchell, M. Ross, R. G. Dandrea, N. W. Ashcroft, N. C.

Holmes, and R. G. Gathers, Phys. Rev. Lett. 60, 1414 (1988).

[37] A. C. Mitchell et al, J. Appl. Phys., 69, 2981 (1991).

[38] W. J. Nellis, A. C. Mitchell, A. K. McMahan, J. Appl. Phys., 90, 696 (2001).

[39] D. J. Erskine, W. J. Nellis, Nature, 349, 317 (1991).

[40] D. J. Erskine, W. J. Nellis, J. Appl. Phys., 71, 4882 (1992).

[41] V. N. Zharkov, T. V. Gudkova, in High-Pressure Research: Application to Earth and Planetary Sciences, edited by Y. Syono and M. H. Manghnani (Terra Scientific Publishing, Tokyo, 1992), pp. 393-401.

[42] M. Ross, H. C. Graboske, W. J. Nellis, Phil. Trans. R. Soc. Lond. A,, 303, 303 (1981).

[43] D. J. Stevenson, Rep. Prog. Phys., 46, 555 (1983).

[44] W. J. Nellis, M. Ross, N. C. Holmes, Science, 269, 1249 (1995).

[45] W. J. Nellis, S. T. Weir, A. C. Mitchell, Science, 273, 936 (1996).

[46] W. J. Nellis, J. Planet. Space Sciences, 48, 671 (2000).

[47] W. B. Hubbard et al, Science, 253, 648 (1991).

[48] D. J. Stevenson, E. E. Salpeter, Astrophys. J. Suppl. Ser., 35, 221 (1977).

[49] D. Saumon, G. Chabrier, Phys. Rev. A , 46, 2084 (1992). 
[50] W. R. D. Magro et al, Phys. Rev. Lett.,. 76, 1240 (1996).

[51] W. B. Hubbard, M. S. Marley, Icarus, 78, 102 (1989).

[52] R. Smoluchowski, Astrophys. J., 200, L119 (1975).

[53] D. J. Stevenson, Annu. Rev. Earth Planet. Sci., 10, 257 (1982).

[54] D. C. Hamilton et al, J. Chem. Phys., 887706 (1988).

[55] W. B. Hubbard et al, Science, 253, 648 (1991).

[56] W. J. Nellis, Encyclopedia of Applied Physics 18, 541 (1997).

[57] A. J. Gratz et al, Phys. Chem. Minerals, 19, 267 (1992).

[58] P. S. Fiske et al, Science, 270, 281 (1995).

[59] R. Koch, W. J. Nellis, J. W. Hunter, H. Davidson, T. H. Geballe, Pract. Met., 27, 391 (1990).

[60] W. J. Nellis, Philos. Mag. B, 79, 655 (1999).

[61] S. M. Pollaine, W. J. Nellis, in Science and Technology of High Pressure, edited by M. H. Manghnani, W. J. Nellis, and M. F. Nicol (Universities Press, Hyderabad, 2000), pp.210-211.

[62] W. J. Nellis et al, in High-Pressure Science and Technology-1993, edited by S. C. Schmidt, J. W. Shaner, G. A. Samara, and M. Ross (American Institute of Physics, New York, 1994), pp. 695697.

[63] R. Chau, M. B. Maple, W. J. Nellis, J. Appl. Phys., 79, 9236 (1996).

[64] T. Sekine, Proc. Japan Acad. Ser. B, 68, 95 (1992).

[65] C. S. Yoo et al, Appl. Phys. Lett., 61, 273 (1992).

[66] J. Freim, J. McKittrick, W. J. Nellis, in High-Pressure Science and Technology-1993, edited by S. C. Schmidt, J. W. Shaner, G. A. Samara, and M. Ross (American Institute of Physics, New York, 1994), pp. 1263-1266.

[67] D. J. Benson, W. J. Nellis, Appl. Phys. Lett., 65, 418 (1994).

[68] W. J. Nellis et al, in Shock Waves in Condensed Matter-1987, edited by S. C. Schmidt and N. C. Holmes (Elsevier, New York, 1988), pp. 407- 410. 


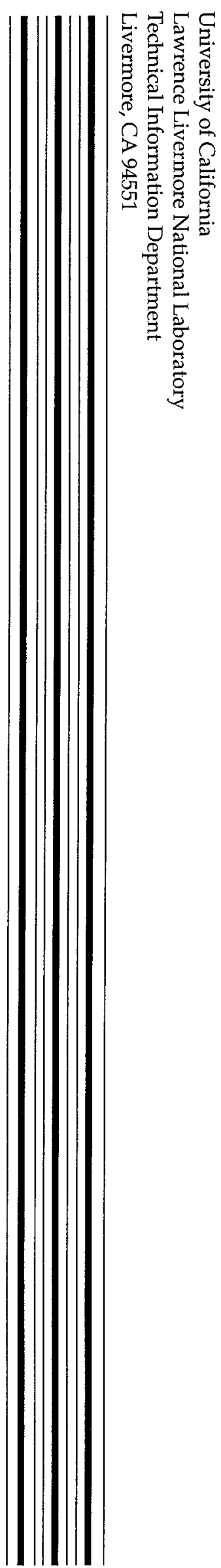

